\title{
Physicochemical properties and thermal behavior of binary blends of Madhuca longifolia seed fat and palm oil as a lard substitute
}

\begin{abstract}
Fat extracted from pork is prohibited under halal and kosher food regulations. A study was carried out on Madhuca longifolia seed fat and palm oil to compare their physicochemical, solidification and melting characteristics to formulate halal alternative lipid substitutes. Various blends of Madhuca longifolia (ML) and palm oil (PO) was formulated in order to become similar to lard (LD). A total of three binary blends were prepared: ML:PO (97:3; w/w), ML:PO (95:5), ML:PO (93:7), and identified by the mass ratio of ML to PO. The fat blends were compared with LD in terms of the fatty acid and triacylglycerol compositions using gas chromatography and high performance liquid chromatography, respectively. In addition, the fat blends also being studied for thermal properties using differential scanning calorimetry and solid fat content using p-nuclear magnetic resonance. Although there were considerable differences between LD and the fat blends with regard to fatty acid and triacylglycerol compositions, some similarities were seen regarding to thermal properties and solid fat content profiles. The blend of ML:PO (97:3) displayed closer similarity to LD with respect to melting transition at $-3.59^{\circ} \mathrm{C}$ and its solid fat content profile showed the least difference to that of LD throughout the temperature range measured.
\end{abstract}

Keyword: Madhuca longifolia; Palm oil; Fatty acid; Triacylglycerol; Thermal behavior; Solid fat content 